\title{
HDL particle size is increased and HDL-cholesterol efflux is enhanced in type 1 diabetes: a cross-sectional study
}

\author{
Mohamad O. Ahmed ${ }^{1} \cdot$ Rachel E. Byrne ${ }^{2} \cdot$ Agnieszka Pazderska $^{1} \cdot$ Ricardo Segurado $^{3} \cdot$ Weili Guo $^{2} \cdot$ Anjuli Gunness $^{1}$. \\ Isolda Frizelle ${ }^{1}$. Mark Sherlock ${ }^{1}$. Khalid S. Ahmed ${ }^{1} \cdot$ Anne McGowan $^{1} \cdot$ Kevin Moore ${ }^{1} \cdot$ Gerard Boran $^{4}$. \\ Fiona C. McGillicuddy ${ }^{2}$. James Gibney ${ }^{1}$
}

Received: 6 May 2020 / Accepted: 9 September 2020 / Published online: 9 November 2020

(C) Springer-Verlag GmbH Germany, part of Springer Nature 2020

\begin{abstract}
Aims/hypothesis The prevalence of atherosclerosis is increased in type 1 diabetes despite normal-to-high HDL-cholesterol levels. The cholesterol efflux capacity (CEC) of HDL is a better predictor of cardiovascular events than static HDL-cholesterol. This cross-sectional study addressed the hypothesis that impaired HDL function contributes to enhanced CVD risk within type 1 diabetes.

Methods We compared HDL particle size and concentration (by NMR), total CEC, ATP-binding cassette subfamily A, member 1 (ABCA1)-dependent CEC and ABCA1-independent CEC (by determining $\left[{ }^{3} \mathrm{H}\right]$ cholesterol efflux from J774-macrophages to ApoB-depleted serum), and carotid intima-media thickness (CIMT) in 100 individuals with type 1 diabetes (37.6 \pm 1.2 years; BMI $\left.26.9 \pm 0.5 \mathrm{~kg} / \mathrm{m}^{2}\right)$ and 100 non-diabetic participants $\left(37.7 \pm 1.1\right.$ years; $\left.27.1 \pm 0.5 \mathrm{~kg} / \mathrm{m}^{2}\right)$.

Results Compared with non-diabetic participants, total HDL particle concentration was lower (mean \pm SD $31.01 \pm 8.66$ vs 34.33 $\pm 8.04 \mu \mathrm{mol} / 1$ [mean difference (MD) $-3.32 \mu \mathrm{mol} / 1]$ ) in participants with type 1 diabetes. However, large HDL particle concentration was greater $(9.36 \pm 3.98 \mathrm{vs} 6.99 \pm 4.05 \mu \mathrm{mol} / 1[\mathrm{MD}+2.37 \mu \mathrm{mol} / 1])$, resulting in increased mean HDL particle size $(9.82 \pm$ 0.57 vs $9.44 \pm 0.56 \mathrm{~nm}[\mathrm{MD}+0.38 \mathrm{~nm}])(p<0.05$ for all $)$. Total CEC $(14.57 \pm 2.47 \% \mathrm{CEC} / 4 \mathrm{~h}$ vs $12.26 \pm 3.81 \% \mathrm{CEC} / 4 \mathrm{~h}[\mathrm{MD}$ $+2.31 \% \mathrm{CEC} / 4 \mathrm{~h}]$ ) was greater in participants with type 1 diabetes relative to non-diabetic participants. Increased HDL particle size was independently associated with increased total CEC; however, following adjustment for this in multivariable analysis, CEC remained greater in participants with type 1 diabetes. Both components of CEC, ABCA1-dependent $(6.10 \pm 2.41 \% \mathrm{CEC} / 4 \mathrm{~h}$ vs $5.22 \pm 2.57 \% \mathrm{CEC} / 4 \mathrm{~h}[\mathrm{MD}+0.88 \% \mathrm{CEC} / 4 \mathrm{~h}])$ and $\mathrm{ABCA} 1$-independent $(8.47 \pm 1.79 \% \mathrm{CEC} / 4 \mathrm{~h}$ vs $7.05 \pm 1.76 \% \mathrm{CEC} / 4 \mathrm{~h}$ $[\mathrm{MD}+1.42 \% \mathrm{CEC} / 4 \mathrm{~h}]) \mathrm{CEC}$, were greater in type 1 diabetes but the increase in ABCA1-dependent CEC was less marked and not statistically significant in multivariable analysis. CIMT was increased in participants with type 1 diabetes but in multivariable analysis it was only associated negatively with age and BMI.

Conclusions/interpretation HDL particle size but not HDL-cholesterol level is independently associated with enhanced total CEC. HDL particle size is greater in individuals with type 1 diabetes but even after adjusting for this, total and ABCA1independent CEC are enhanced in type 1 diabetes. Further studies are needed to understand the mechanisms underlying these effects, and whether they help attenuate progression of atherosclerosis in this high-risk group.
\end{abstract}

Keywords HDL particle size $\cdot$ HDL-cholesterol efflux capacity · Type 1 diabetes

Mohamad O. Ahmed and Rachel E. Byrne are equal contributors. James Gibney and Fiona C. McGillicuddy share senior authorship.

James Gibney

James.Gibney@TUH.ie

1 Robert Graves Institute of Endocrinology, Tallaght University Hospital, Dublin, Ireland

2 Diabetes Complications Research Centre, School of Medicine, University College Dublin, Belfield, Dublin, Ireland
3 School of Public Health, Physiotherapy, and Sports Science, University College Dublin, Belfield, Dublin, Ireland

4 Department of Chemical Pathology, Tallaght University Hospital, Dublin, Ireland 


\section{Research in context}

\section{What is already known about this subject?}

- Increased atherosclerotic disease in type 1 diabetes is not fully explained by traditional cardiovascular risk factors

- HDL-cholesterol efflux capacity (CEC) is a better predictor of cardiovascular outcome than HDL-cholesterol levels

- HDL-cholesterol levels are increased in type 1 diabetes but there is some evidence that this increase is not associated with improved $\mathrm{HDL}$ function

What is the key question?

- Does HDL CEC differ in individuals with type 1 diabetes compared with similar people without diabetes, and are any observed changes related to HDL particle size?

\section{What are the new findings?}

- HDL particle size, but not HDL-cholesterol level, is independently associated with enhanced total CEC. HDL particle size is greater in type 1 diabetes but, even after adjusting for this, total and particularly ATP-binding cassette subfamily $A$, member 1 (ABCA1)-independent CEC are enhanced in type 1 diabetes

How might this impact on clinical practice in the foreseeable future?

- Measurements of HDL particle size and function might be more useful than HDL-cholesterol levels in evaluating cardiovascular risk in type 1 diabetes

$\begin{array}{ll}\text { Abbreviations } \\ \text { ABCA1 } & \text { ATP-binding cassette subfamily A, member 1 } \\ \text { ABCG1 } & \text { ATP-binding cassette subfamily G, member 1 } \\ \text { CEC } & \text { Cholesterol efflux capacity } \\ \text { CETP } & \text { Cholesteryl ester transfer protein } \\ \text { CIMT } & \text { Carotid intima-media thickness } \\ \text { JUPITER } & \text { Justification for the Use of Statins in Prevention: } \\ & \text { an Intervention Trial Evaluating Rosuvastatin } \\ \text { MACE } & \text { Major atherosclerotic cardiac events } \\ \text { RCT } & \text { Reverse cholesterol transport } \\ \text { SAA } & \text { Serum amyloid A }\end{array}$

\section{Introduction}

Despite many improvements in the management of type 1 diabetes, life expectancy for affected individuals remains approximately 12 years less than for the non-diabetic population $[1,2]$. This is mostly attributable to an elevated risk of CVD that is not explained by conventional risk factors. In contrast to individuals with type 2 diabetes, lipid profiles in type 1 diabetes are normal or even apparently better than in the general population, with higher HDL-cholesterol, lower LDLcholesterol and lower triacylglycerol levels [3]. However, these relatively simple lipid measurements potentially mask more subtle lipoprotein abnormalities, including disorders of lipoprotein function, that might contribute to residual atherosclerosis risk in type 1 diabetes.
The importance of a more complete understanding of HDL biology is emphasised by the observation that although HDLcholesterol levels are inversely associated with CVD [4], increasing HDL-cholesterol levels using cholesteryl ester transfer protein (CETP) inhibitors was found not to result in clinical benefits in high-risk individuals $[5,6]$. HDL particles exert numerous atheroprotective functions including antioxidant, anti-inflammatory and cholesterol efflux promoting actions [7]. HDL particles play a critical role in the reverse cholesterol transport (RCT) pathway by promoting efflux of cholesterol from peripheral cells, including lipid-laden foam cells within atherosclerotic lesions, and delivering this acquired lipid back to the liver [8]. Non-esterified cholesterol can be shunted from the liver to the gallbladder via the ABCG5/8 heterodimer [9] or converted into bile acids and shuttled to the gallbladder via ABCB11 [10]. Macrophages efflux cholesterol to smaller HDL particles via the ATPbinding cassette subfamily A, member 1 (ABCA1) transporter while larger particles interact with ATP-binding cassette subfamily G, member 1 (ABCG1) and scavenger receptor class B member 1 (SR-B1) transporters [11]. Evidence that the potentially atheroprotective effects of HDL are clinically relevant is provided by a significant body of literature demonstrating cross-sectional and longitudinal associations between the cholesterol efflux capacity (CEC) of HDL and evidence of both atherosclerosis and cardiovascular events [12-14].

There are theoretical reasons why HDL function in individuals with type 1 diabetes might differ from that in nondiabetic individuals [3]. These include greater particle size, 
reduced HDL triacylglycerol content, reduced cholesterol esters in small HDL3 particles, increased susceptibility to oxidation, increases in phospholipid transfer protein (PLTP), CETP and lecithin-cholesterol acyltransferase (LCAT), and changes in the HDL proteome (summarised in detail in [15]). Data from the Pittsburgh Epidemiology of Diabetes Complications Study demonstrate that the usual inverse association between HDL-cholesterol and coronary artery disease risk, although retained in men, is altered in women with type 1 diabetes; the women show little increased protection with concentrations above the range of $50-60 \mathrm{mg} / \mathrm{dl}(1.3-$ $1.55 \mathrm{mmol} / \mathrm{l}$ ), suggesting that at least in some individuals, HDL is dysfunctional [16]. Few studies to date have addressed HDL function in type 1 diabetes and none relate aspects of HDL function to early evidence of atherosclerosis.

The aims of this study were to compare total CEC, ABCA1-dependent CEC and ABCA1-independent CEC between individuals with type 1 diabetes and matched control participants to determine whether any observed between-group differences were associated with differences in HDL particle size. We also aimed to determine whether HDL particle size and/or CEC were associated with carotid intima-media thickness (CIMT), a marker of early atherosclerosis.

\section{Methods}

Study design and participants This was a cross-sectional study designed to compare HDL particle concentration, HDL particle size and CEC between 100 participants with type 1 diabetes and 100 non-diabetic participants matched for age \pm 5 years, sex and BMI $\pm 3 \mathrm{~kg} / \mathrm{m}^{2}$.

Participants with type 1 diabetes were contacted either by phone or at the time of their scheduled clinic visit. Inclusion criteria were as follows: type 1 diabetes; duration of diabetes of at least 1 year; age $18-69$ years; BMI $18-40 \mathrm{~kg} / \mathrm{m}^{2}$; and $\mathrm{HbA}_{1 \mathrm{c}} \leq 97 \mathrm{mmol} / \mathrm{mol}(\leq 11 \%)$. Exclusion criteria were as follows: non-white ethnicity; pregnant or lactating; and recent illness or any chronic illness likely to influence results. Each participant with type 1 diabetes was pair-matched with a healthy volunteer on no medications, recruited from the general population. Inclusion criteria were as follows: non-diabetic; age 18-69 years; and BMI $18-40 \mathrm{~kg} / \mathrm{m}^{2}$. Exclusion criteria were as for participants with type 1 diabetes. Twenty-seven participants with type 1 diabetes and no non-diabetic participants were on statin treatment.

Ethical approval All participants gave written informed consent to the study, which was approved by the Research Ethics Committee of the Adelaide and Meath Hospital and St James' Hospital (Dublin, Ireland).
Study protocol and anthropometric data All participants were studied after they had fasted for $12 \mathrm{~h}$, having avoided excessive exercise and alcohol for the previous $24 \mathrm{~h}$. Height was measured with a Harpenden stadiometer. Weight was measured in a hospital gown. Data relating to previous medical history, medication use and smoking status were recorded. Alcohol consumption was recorded in all participants with type 1 diabetes and in 80 of 100 non-diabetic participants.

Laboratory methods Plasma levels of total cholesterol, triacylglycerols and HDL-cholesterol were measured by an enzymatic calorimetric method on the Roche P Module (Roche, Mannheim, Germany). Additional samples were centrifuged at $1500 \mathrm{~g}$ for $15 \mathrm{~min}$ at $4^{\circ} \mathrm{C}$, and plasma and serum were stored at $-80^{\circ} \mathrm{C}$ until the end of the study.

CEC J774 murine macrophages, derived from BALB/c mice (European Collection of Authenticated Cell Cultures), were seeded $\left(7 \times 10^{5}\right.$ cells $\left./ \mathrm{ml}\right)$ onto 96 -well plates for $24 \mathrm{~h}$, and cultured at $37^{\circ} \mathrm{C}$. Cells were subsequently labelled with $\left[{ }^{3} \mathrm{H}\right]$ cholesterol $(1 \mu \mathrm{Ci} / \mathrm{ml})$ for $24 \mathrm{~h}$ before being equilibrated for $18 \mathrm{~h}$ in DMEM containing 0.2\% BSA with or without cAMP $(0.3 \mathrm{mmol} / \mathrm{l})$ to drive ABCA1 expression. J774 macrophages do not express ABCA1 basally, and stimulation with cAMP specifically drives ABCA1 protein expression. ApoBcontaining lipoproteins were removed from serum by polyethylene glycol precipitation, leaving an HDL-enriched supernatant fraction. Ex vivo efflux from labelled macrophages to $2.8 \%$ HDL-enriched supernatant fraction in minimal essential media (MEM) was measured over $4 \mathrm{~h}$. Percentage efflux was determined by liquid scintillation counting. The difference in efflux to HDL from cells stimulated in the presence and absence of cAMP was taken to represent ABCA1-dependent CEC while ABCA1-independent CEC was calculated from unstimulated cells.

NMR HDL particle concentration and size were measured in serum samples using $400 \mathrm{MHZ}$ NMR spectroscopy (LipoScience, Raleigh, NC, USA). Each lipoprotein subclass emits a distinctive lipid methyl group NMR signal and the amplitudes of the measured signals are directly proportional to the number of subclass particles, which in turn provides the estimated $\mathrm{mol} / \mathrm{l}$ concentration for the three HDL particle subclasses (large 8.9-13 nm, medium 8.3-8.8 nm, small 7.3-8.2 nm) [17]. Weighted-average HDL particle sizes in nanometres were calculated from the subclass levels, and the diameters were assigned to each subclass.

CIMT CIMT was measured as previously described [18] in all participants using a single ultrasonographic machine (Sonosite Micromaxx Ultrasound System, Sonosite, Japan). Measurements were taken from each common carotid artery, 
within $1 \mathrm{~cm}$ of the carotid bulb, with three views of each artery measured, and averaged to form a composite measurement.

Statistical analysis Results in tables are presented as mean \pm SD unless otherwise specified. Data in graphs are presented as mean \pm SD. Data were tested for normality using the ShapiroWilk test, as well as inspected visually using histograms. Statistical significance for categorical variables was calculated using the $\chi^{2}$ test. For continuous variables, the paired Student's $t$ test was used to assess statistical significance. Correlations were evaluated using the Pearson correlation or Spearman test as appropriate. Generalised estimating equations with robust Huber-White (sandwich) SE estimation was used to examine the joint effect of all predictors while accounting for the non-independence of matched pairs. IBM SPSS Statistics (Version 24.0. Armonk, NY) and GraphPad Prism (Version 8.4.3. San Diego, CA) software were used for data analysis. Statistical significance is presented as $p<0.05$, $p<0.01$ and $p<0.001$.

\section{Results}

Clinical and biochemical characteristics of 100 participants with type 1 diabetes and 100 healthy non-diabetic participants are shown in Table 1. There were no significant differences in total cholesterol or triacylglycerol levels between type 1 diabetes and control groups. LDL-cholesterol was lower, HDL-cholesterol and $\mathrm{HbA}_{1 \mathrm{c}}$ were higher, and CIMT was greater in participants with type 1 diabetes than in non- diabetic participants (Table 1, Fig. 1). Mean alcohol consumption was greater in the non-diabetic participants, although the difference did not reach statistical significance.

HDL particle size and subclass distribution Total HDL particle concentration was significantly lower in participants with type 1 diabetes (Fig. 1a) than in non-diabetic participants despite HDL-cholesterol concentrations being higher (Table 1). This reduction was specifically evident in women (mean \pm SD for type 1 diabetes vs non-diabetic group: $28.42 \pm 9.64$ vs 34.91 $\pm 9.22 \mu \mathrm{mol} / \mathrm{l}$ [mean difference $-6.49 \mu \mathrm{mol} / 1]$ ) with no effect evident in men $(34.79 \pm 5.07$ vs $33.77 \pm 5.99 \mu \mathrm{mol} / 1$ [mean difference $1.02 \mu \mathrm{mol} / 1]$ ) (Fig. 1a). Mean HDL particle size was greater in participants with type 1 diabetes than in nondiabetic participants, with similar effects evident in men and women (Fig. 1b). Consistent with an increase in mean HDL particle size, the concentration of large HDL particles was increased in participants with type 1 diabetes, with similar patterns evident in men $(7.09 \pm 3.34$ vs $4.56 \pm 2.15 \mu \mathrm{mol} / 1$ [mean difference $+2.53 \mu \mathrm{mol} / \mathrm{l}])$ and women $(10.92 \pm 3.64$ vs $8.68 \pm 4.25 \mu \mathrm{mol} / 1$ [mean difference $+2.24 \mu \mathrm{mol} / 1$ ]) (Fig. 1c). By contrast, the concentration of medium and small HDL particles was significantly reduced in participants with type 1 diabetes relative to non-diabetic participants (Fig. 1d,e). These effects were particularly pronounced in women: mean $\pm \mathrm{SD}$ HDL particle concentration for type 1 diabetes vs nondiabetic groups was $10.08 \pm 6.91$ vs $13.64 \pm 7.77 \mu \mathrm{mol} / 1$ (mean difference $-3.56 \mu \mathrm{mol} / 1, p>0.05$ ) for medium HDL particles and $7.44 \pm 5.77$ vs $12.53 \pm 7.03 \mu \mathrm{mol} / \mathrm{l}$ (mean difference $-5.09 \mu \mathrm{mol} / 1, p<0.001$ ) for small HDL particles. A non-

Table 1 Baseline characteristics of type 1 diabetes and non-diabetic cohorts

\begin{tabular}{|c|c|c|c|c|c|}
\hline Characteristic & $\mathrm{T} 1 \mathrm{DM}(n=100)$ & Non-diabetic $(n=100)$ & Mean difference & $95 \% \mathrm{CI}$ & $p$ value \\
\hline Sex, $n$ male $/ n$ female & $40 / 60$ & $40 / 60$ & NA & NA & 1.0 \\
\hline Age, years & $37.6 \pm 11.9$ & $37.7 \pm 11.0$ & -0.1 & $-0.6,0.5$ & 0.8 \\
\hline BMI, $\mathrm{kg} / \mathrm{m}^{2}$ & $26.9 \pm 4.9$ & $27.1 \pm 5.0$ & -0.2 & $-0.4,0.1$ & 0.13 \\
\hline Smoker, \% & 20 & 18 & N/A & N/A & 0.68 \\
\hline Alcohol intake, units/week & $2(0-8)$ & $6(0-12)$ & N/A & $-4.9,-0.1$ & $0.04 *$ \\
\hline Systolic BP, mmHg & $128 \pm 16$ & $127 \pm 15$ & 1 & $-3,5$ & 0.58 \\
\hline Diastolic BP, mmHg & $77 \pm 9$ & $82 \pm 11$ & -6 & $-8,-3$ & $<0.0001 * * *$ \\
\hline CIMT, mm & $0.590 \pm 0.084$ & $0.556 \pm 0.071$ & 0.034 & $0.003,0.047$ & $0.03 *$ \\
\hline $\mathrm{HbA}_{1 \mathrm{c}}, \mathrm{mmol} / \mathrm{mol}$ & $70 \pm 16$ & $35 \pm 3$ & 35 & 32,39 & $<0.0001 * * *$ \\
\hline $\mathrm{HbA}_{1 \mathrm{c}}, \%$ & $8.5 \pm 1.5$ & $5.3 \pm 0.3$ & 3.2 & $2.9,3.6$ & $<0.0001 * * *$ \\
\hline Total cholesterol concentration, mmol/1 & $4.5 \pm 0.9$ & $4.7 \pm 0.9$ & -0.2 & $-0.4,0.1$ & 0.32 \\
\hline Triacylglycerol concentration, $\mathrm{mmol} / 1$ & $0.9(0.7-1.3)$ & $0.9(0.7-1.3)$ & N/A & $-0.1,0.3$ & 0.37 \\
\hline LDL-cholesterol concentration, mmol/1 & $2.3 \pm 0.9$ & $2.7 \pm 0.8$ & -0.4 & $-0.6,-0.1$ & $0.004 * *$ \\
\hline HDL-cholesterol concentration, mmol/1 & $1.6 \pm 0.4$ & $1.5 \pm 0.4$ & 0.1 & $0.1,0.3$ & $0.002 * *$ \\
\hline
\end{tabular}

Data are displayed as mean $\pm \mathrm{SD}$, median (IQR) or \%

$* p<0.05, * * p<0.01$ and $* * * p<0.001$ for type 1 diabetes vs non-diabetic cohort

T1DM, type 1 diabetes 
Fig. 1 NMR analysis of HDL particles in individuals with type 1 diabetes and non-diabetic participants (control), showing results for total cohort combined $(n=100)$ and separately for men $(n=40)$ and women $(n=60)$. Bar charts show total HDL particle concentration (a), mean HDL particle diameter (b), large HDL particle concentration (c), medium HDL particle concentration (d) and small HDL particle concentration (e). Data are presented as mean $\pm \mathrm{SD}$. $* p<0.05, * * p<0.01 * * * p<0.001$ for type 1 diabetes vs non-diabetic control. HDL-P, HDL particle; T1D, type 1 diabetes
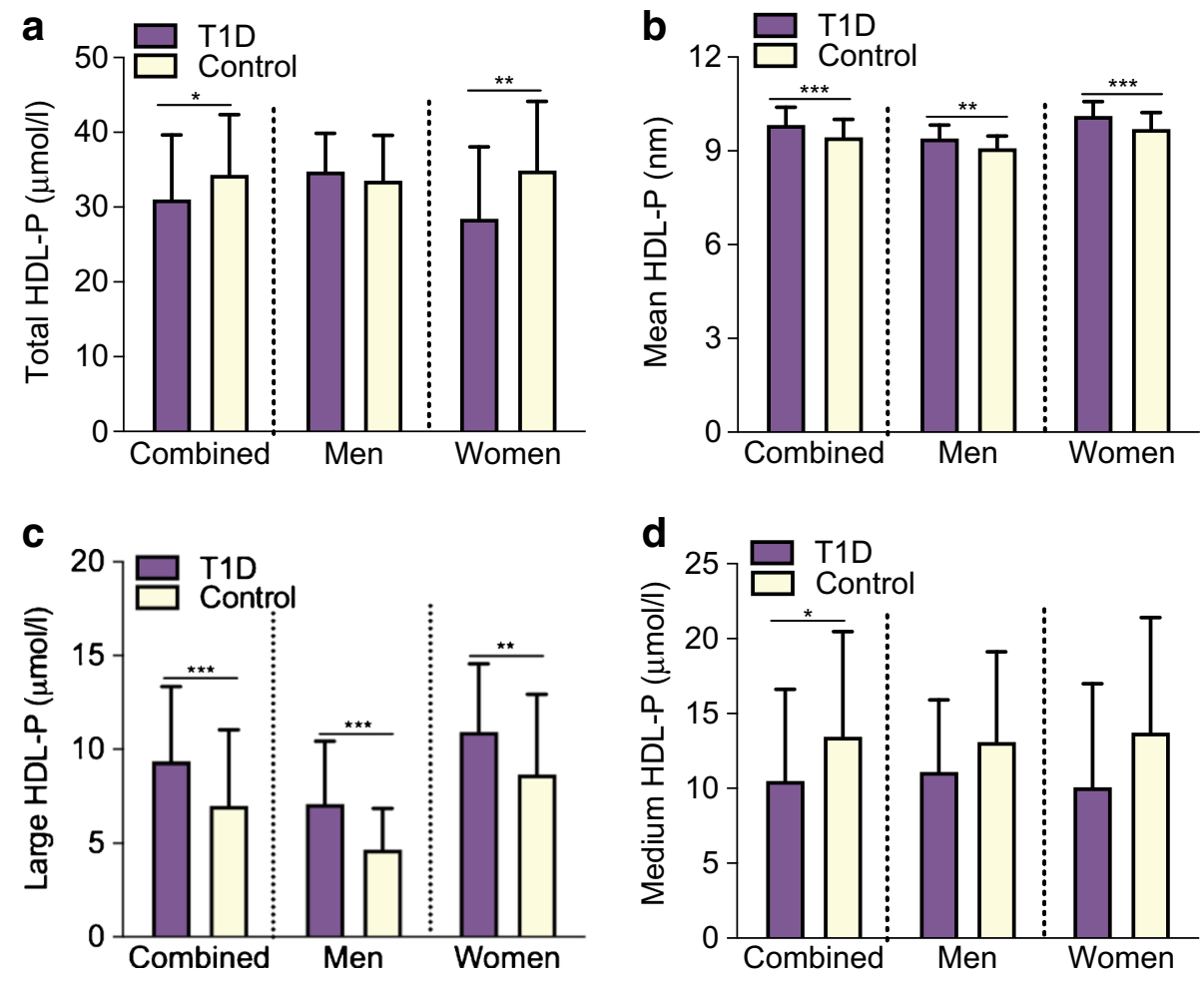

significant reduction in medium HDL particle concentration $(11.1 \pm 4.8$ vs $13.11 \pm 6.12 \mu \mathrm{mol} / 1$ [mean difference $-2.01 \mu \mathrm{mol} / \mathrm{l}]$ ), and a non-significant increase in small HDL particle concentration $(16.6 \pm 4.95$ vs $16.1 \pm 5.66 \mu \mathrm{mol} / 1$ [mean difference $+0.5 \mu \mathrm{mol} / 1]$ ) was evident in men with type 1 diabetes relative to non-diabetic participants (Fig. 1d,e).

HDL CEC Total CEC was significantly greater in participants with type 1 diabetes than in non-diabetic participants (Fig. 2a, Table 2). A significant increase in both ABCA1-dependent and ABCA1-independent efflux was evident in participants with type 1 diabetes relative to non-diabetic participants (Fig. 2b,c). Interestingly, total CEC and ABCA1independent $\mathrm{CEC}$ were increased to a greater extent relative to non-diabetic participants in men $(14.48 \pm 2.51 \% \mathrm{CEC} / 4 \mathrm{~h}$ vs $11.02 \pm 4.03 \% \mathrm{CEC} / 4 \mathrm{~h}$ [mean difference $+3.46 \% \mathrm{CEC} / 4 \mathrm{~h}$ ] and $9.16 \pm 1.65 \% \mathrm{CEC} / 4 \mathrm{~h}$ vs $6.43 \pm 2.06 \% \mathrm{CEC} / 4 \mathrm{~h}$ [mean difference $+2.73 \% \mathrm{CEC} / 4 \mathrm{~h}$ ], respectively) than in women $(14.63 \pm 2.46 \% \mathrm{CEC} / 4 \mathrm{~h}$ vs $13.16 \pm 3.46 \% \mathrm{CEC} / 4 \mathrm{~h}$ [mean difference $+1.47 \% \mathrm{CEC} / 4 \mathrm{~h}]$ and $8.01 \pm 1.74 \% \mathrm{CEC} / 4 \mathrm{~h}$ vs $7.44 \pm 1.42 \% \mathrm{CEC} / 4 \mathrm{~h}$ [mean difference $+0.57 \% \mathrm{CEC} / 4 \mathrm{~h}$ ], respectively) (Fig. 2a,c). In parallel, CIMT burden was a significantly greater in men with type 1 diabetes relative to non-diabetic men $(0.618 \pm 0.09$ vs $0.566 \pm 0.07 \mathrm{~mm}$ [mean difference $+0.052 \mathrm{~mm}], p<0.01$ ) with no significant difference evident in women (Fig. 2d).

Effect of alcohol consumption To ensure the observed differences were not due to the finding that mean alcohol consumption was (non-significantly) greater in the non-diabetic individuals, comparisons were repeated in participants with an alcohol intake $\leq 10$ units/week (79/100 participants with type 1 diabetes; 59/80 non-diabetic participants). There was no difference in findings (mean \pm SD values for participants with 
Table 2 CEC and lipoprotein particle analysis in type 1 diabetes and non-diabetic cohorts

\begin{tabular}{lcccr}
\hline Variable & T1DM $(n=100)$ & Non-diabetic $(n=100)$ & Mean difference & $95 \%$ CI \\
\hline Total CEC, \%CEC/4 h & $14.57 \pm 2.47$ & $12.26 \pm 3.81$ & +2.31 & $1.36,3.35 * * *$ \\
ABCA1-dependent CEC, \%CEC/4 h & $6.10 \pm 2.41$ & $5.22 \pm 2.57$ & +0.88 & $0.19,1.66 *$ \\
ABCA1-independent CEC, \%CEC/4 h & $8.47 \pm 1.79$ & $7.05 \pm 1.76$ & +1.42 & $0.90,1.97 * * *$ \\
Mean HDL-P size, nm & $9.82 \pm 0.57$ & $9.44 \pm 0.56$ & +0.38 & $0.24,0.49 * * *$ \\
Total HDL-P concentration, $\mu \mathrm{mol} / 1$ & $31.01 \pm 8.66$ & $34.33 \pm 8.04$ & -3.32 & $-5.57,-0.29 *$ \\
Large HDL-P concentration, $\mu \mathrm{mol} / 1$ & $9.36 \pm 3.98$ & $6.99 \pm 4.05$ & -2.96 & $1.48,3.41 * * *$ \\
Medium HDL-P concentration, $\mu \mathrm{mol} / 1$ & $10.49 \pm 6.13$ & $13.45 \pm 7.02$ & -2.74 & $-5.01,-0.48 *$ \\
Small HDL-P concentration, $\mu \mathrm{mol} / 1$ & $11.16 \pm 7.06$ & $13.90 \pm 6.7$ & $-4.63,-0.62 *$ \\
\hline
\end{tabular}

Data are displayed as mean $\pm \mathrm{SD}$

${ }^{*} p<0.05$ and $* * * p<0.001$ for type 1 diabetes vs non-diabetic cohort

HDL-P, HDL particle; T1DM, type 1 diabetes

type 1 diabetes vs non-diabetic participants: HDL particle size $9.84 \pm 0.59$ vs $9.43 \pm 0.55 \mathrm{~nm}, p<0.0005$; total CEC $14.52 \pm$ $2.49 \% \mathrm{CEC} / 4 \mathrm{~h}$ vs $12.32 \pm 4.01 \% \mathrm{CEC} / 4 \mathrm{~h} p<0.0005$; ABCA1-dependent CEC $6.19 \pm 2.47 \% \mathrm{CEC} / 4 \mathrm{~h}$ vs $5.24 \pm$ 2.70\%CEC/4 h, $p<0.05$; ABCA1-independent CEC $8.32 \pm$ $1.84 \% \mathrm{CEC} / 4 \mathrm{~h}$ vs $7.07 \pm 1.83 \% \mathrm{CEC} / 4 \mathrm{~h}, p<0.0005)$.

Univariable and multivariable associations In univariable analysis, total CEC was positively associated with type 1 diabetes status (greater in type 1 diabetes vs no diabetes), total cholesterol concentration and HDL particle size, and negatively associated with age (Table 3). In multivariable analysis, total CEC was positively associated with type 1 diabetes status, total cholesterol concentration, HDL particle size and statin treatment (greater with vs without statin treatment), and negatively associated with age (Table 3 ).

In univariable analysis, ABCA1-dependent CEC was positively associated with total cholesterol and triacylglycerol concentration (Table 4). In multivariable analysis, ABCA1-dependent CEC was positively associated with total cholesterol concentration and negatively associated with age (Table 4).

In both univariable and multivariable analysis, ABCA1independent $\mathrm{CEC}$ was positively associated with type 1 diabetes status, HDL particle concentration and statin treatment (Table 5).
Fig. 2 HDL CEC and CIMT measurements in participants with type 1 diabetes and non-diabetic participants (control), showing results for total cohort combined $(n=100)$ and separately for men $(n=40)$ and women $(n=60)$. Bar charts show total CEC (a), ABCA1-dependent CEC (b), ABCA1-independent CEC (c) and CIMT measurements (d). Data are presented as mean $\pm \mathrm{SD}$. Statistical significance is presented as $* p<0.05, * * p<0.01$ and $* * * p<0.001$ for type 1 diabetes vs non-diabetic control. T1D, type 1 diabetes
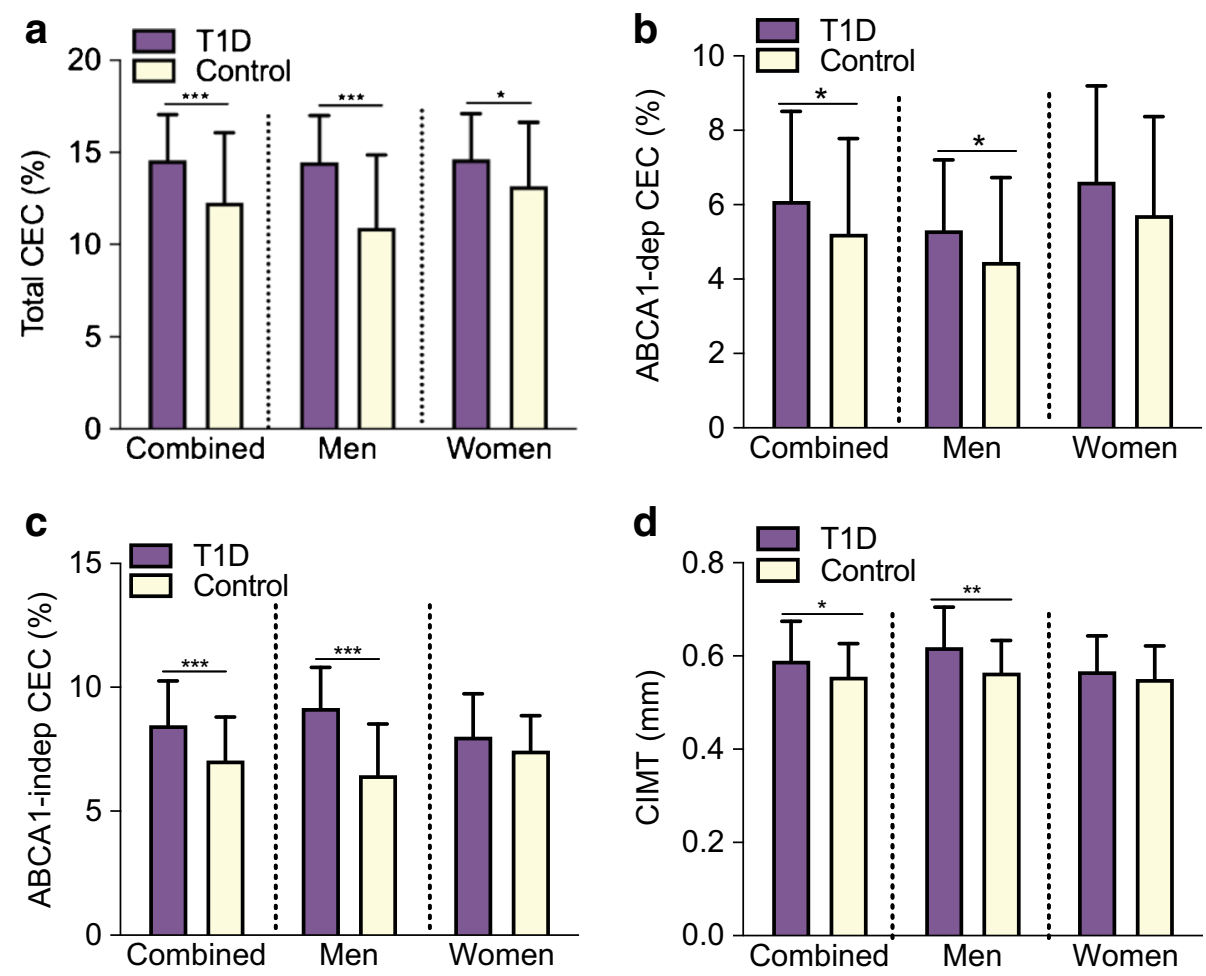
Table 3 Univariable and multivariable association of clinical and biochemical variables with HDL CEC

\begin{tabular}{|c|c|c|c|c|}
\hline \multirow[t]{2}{*}{ Variable } & \multicolumn{2}{|c|}{ Univariable model } & \multicolumn{2}{|c|}{ Multivariable model } \\
\hline & $\beta$ coefficient & $95 \% \mathrm{CI}$ & $\beta$ coefficient & $95 \% \mathrm{CI}$ \\
\hline Age (years) & -0.061 & $-0.116,-0.007$ & -0.061 & $-0.114,-0.008$ \\
\hline Sex (1 male, 2 female) & 0.540 & $-0.696,1.776$ & 0.624 & $-1.882,0.634$ \\
\hline $\operatorname{BMI}\left(\mathrm{kg} / \mathrm{m}^{2}\right)$ & 0.006 & $-0.094,0.106$ & 0.002 & $-0.082,0.085$ \\
\hline Type 1 diabetes ( 1 no, 2 yes) & 1.507 & $0.318,2.695$ & 1.465 & $0.265,2.666$ \\
\hline Total cholesterol (mmol/l) & 0.885 & $0.195,1.576$ & 0.870 & $0.195,1.545$ \\
\hline Triacylglycerols (mmol/l) & 0.586 & $-0.249,1.421$ & 0.629 & $-0.351,1.608$ \\
\hline HDL-cholesterol (mmol/l) & 0.804 & $-1.581,3.189$ & 0.600 & $-2.206,3.406$ \\
\hline HDL-P concentration $(\mu \mathrm{mol} / \mathrm{l})$ & 0.055 & $-0.033,0.144$ & 0.050 & $-0.039,0.138$ \\
\hline HDL-P size (nm) & 2.426 & $0.442,4.409$ & 2.173 & $0.183,4.163$ \\
\hline Large HDL-P concentration $(\mu \mathrm{mol} / \mathrm{l})$ & -0.189 & $-0.477,0.098$ & -0.142 & $-0.420,0.136$ \\
\hline Statin treatment ( 1 no, 2 yes $)$ & 1.988 & $-0.012,3.988$ & 2.093 & $0.625,3.561$ \\
\hline
\end{tabular}

$\beta$ coefficient was derived from linear regression and generalised estimating equation

HDL-P, HDL particle

In univariable analysis, CIMT was positively associated with ABCA1-dependent CEC and ABCA1-independent $\mathrm{CEC}$ and negatively associated with age and BMI (data not shown). In multivariable analysis, CIMT was negatively associated with age and BMI.

\section{Discussion}

We have demonstrated reduced total HDL particle concentration in type 1 diabetes, but greater large HDL particle concentration, resulting in greater mean HDL particle size. Increased HDL particle size was independently associated with increased total CEC; however, even following adjustment for increased HDL-cholesterol and HDL particle size in multivariable analysis, CEC remained greater in participants with type 1 diabetes compared with closely matched participants without diabetes. Both components of CEC, ABCA1dependent and $\mathrm{ABCA} 1$-independent $\mathrm{CEC}$, were greater in type 1 diabetes but the increase in ABCA1-dependent CEC was less marked and not statistically significant in multivariable analysis.

This is the first study in type 1 diabetes to determine ABCA1-dependent and ABCA1-independent contributions to $\mathrm{CEC}$, and the first to address associations between CEC, HDL particle size and HDL particle concentration, and
Table 4 Univariable and multivariable association of clinical and biochemical variables with ABCA1-dependent CEC in participants with type 1 diabetes and participants without diabetes

\begin{tabular}{|c|c|c|c|c|}
\hline \multirow[t]{2}{*}{ Variable } & \multicolumn{2}{|c|}{ Univariable model } & \multicolumn{2}{|c|}{ Multivariable model } \\
\hline & $\beta$ coefficient & $95 \% \mathrm{CI}$ & $\beta$ coefficient & $95 \% \mathrm{CI}$ \\
\hline Age (years) & -0.040 & $-0.081,0.001$ & -0.041 & $-0.077,-0.004$ \\
\hline Sex (1 male, 2 female) & 0.731 & $-0.201,1.664$ & 0.827 & $-1.745,0.092$ \\
\hline BMI $\left(\mathrm{kg} / \mathrm{m}^{2}\right)$ & -0.011 & $-0.086,0.65$ & -0.017 & $-0.086,0.053$ \\
\hline Type 1 diabetes (1 no, 2 yes) & 0.073 & $-0.824,0.969$ & 0.078 & $-0.965,0.810$ \\
\hline Total cholesterol concentration (mmol/l) & 0.700 & $0.179,1.221$ & 0.686 & $0.151,1.222$ \\
\hline Triacylglycerol concentration (mmol/l) & 0.698 & $0.068,1.328$ & 0.739 & $-0.036,1.515$ \\
\hline HDL-cholesterol concentration (mmol/l) & 0.572 & $-1.227,2.371$ & 0.432 & $-1.974,2.838$ \\
\hline HDL-P concentration $(\mu \mathrm{mol} / \mathrm{l})$ & -0.055 & $-0.121,0.012$ & -0.056 & $-0.125,0.012$ \\
\hline HDL-P size (nm) & 1.447 & $-0.049,2.944$ & 1.349 & $-0.184,2.882$ \\
\hline Large HDL-P concentration $(\mu \mathrm{mol} / \mathrm{l})$ & -0.119 & $-0.336,0.097$ & -0.108 & $-0.336,0.121$ \\
\hline Statin treatment (1 no, 2 yes) & 0.887 & $-0.622,2.396$ & 0.973 & $-2.086,0.140$ \\
\hline
\end{tabular}

$\beta$ coefficient derived from linear regression and generalised estimating equation HDL-P, HDL particle 
Table 5 Univariable and multivariable association of clinical and biochemical variables with ABCA1-independent CEC in participants with type 1 diabetes and participants without diabetes

\begin{tabular}{|c|c|c|c|c|}
\hline \multirow[t]{2}{*}{ Variable } & \multicolumn{2}{|c|}{ Univariable model } & \multicolumn{2}{|l|}{ Multivariable model } \\
\hline & $\beta$ coefficient & $95 \% \mathrm{CI}$ & $\beta$ coefficient & $95 \% \mathrm{CI}$ \\
\hline Age (years) & -0.021 & $-0.049,0.007$ & -0.021 & $-0.046,0.004$ \\
\hline Sex (1 male, 2 female) & -0.191 & $-0.823,0.441$ & -0.198 & $-0.384,0.781$ \\
\hline $\operatorname{BMI}\left(\mathrm{kg} / \mathrm{m}^{2}\right)$ & 0.017 & $-0.035,0.068$ & 0.017 & $-0.026,0.061$ \\
\hline Type 1 diabetes ( 1 no, 2 yes) & 1.434 & $0.827,2.041$ & 1.420 & $0.835,2.004$ \\
\hline Total cholesterol concentration (mmol/l) & 0.185 & $-0.168,0.007$ & 0.186 & $-0.155,0.527$ \\
\hline Triacylglycerol concentration $(\mathrm{mmol} / \mathrm{l})$ & -0.112 & $-0.539,0.314$ & -0.115 & $-0.529,0.299$ \\
\hline HDL-cholesterol concentration (mmol/l) & 0.233 & $-0.986,1.452$ & 0.215 & $-1.001,1.431$ \\
\hline HDL-P concentration $(\mu \mathrm{mol} / \mathrm{l})$ & 0.110 & $0.065,0.155$ & 0.109 & $0.069,0.149$ \\
\hline HDL-P size (nm) & 0.975 & $-0.039,1.989$ & 0.927 & $-0.046,1.899$ \\
\hline Large HDL-P concentration $(\mu \mathrm{mol} / \mathrm{l})$ & -0.070 & $-0.217,2.041$ & -0.058 & $-0.202,0.086$ \\
\hline Statin treatment ( 1 no, 2 yes $)$ & 1.102 & $0.079,2.124$ & 1.103 & $0.213,1.993$ \\
\hline
\end{tabular}

$\beta$ coefficient was derived from linear regression and generalised estimating equation

HDL-P, HDL particle

evidence of early atherosclerosis (CIMT). The study includes the following strengths: (1) comparison between a cohort of 100 type 1 diabetic participants and carefully matched nondiabetic participants; (2) profiling of HDL particle size by NMR and corresponding measurement of particle CEC; and (3) measurement of CIMT, an established marker of early atherosclerosis. Limitations include the fact that this was a cross-sectional study and was not designed to determine the longitudinal effect of the measured variables on progression of atherosclerosis and major atherosclerotic cardiac events (MACE). Furthermore, the study was performed within a population of white ethnicity; it is likely that the variables tested will be influenced by ethnicity, which should be an important consideration for future studies. Finally measures of HDL CEC do not capture information regarding the influence of type 1 diabetes on macrophage/cellular efflux capacity; Daffu et al [19] showed previously that murine diabetic macrophages exhibit impaired ABCG1-mediated efflux in an AGE receptor-dependent mechanism while Mauldin et al [20] similarly demonstrated that monocyte-derived macrophages from individuals with type 2 diabetes exhibit reduced CEC. To date, little is known about macrophage efflux capacity in type 1 diabetes.

Most of the studies on HDL-cholesterol levels in type 1 diabetes reported a significant increase in levels compared with non-diabetic control populations (summarised in [15]). Previous smaller studies investigating HDL function in type 1 diabetes have reported conflicting findings. De Vries et al demonstrated increased total CEC in 14 individuals with type 1 diabetes compared with healthy controls [21]. In contrast, Manjunatha et al demonstrated reduced HDL CEC in individuals with type 1 diabetes who had poor glycaemic control and good glycaemic control (15 in each group), relative to matched non-diabetic counterparts [22]; notably differences in CEC were small and plasma HDL-cholesterol concentration was equivalent across groups. The current study confirms that CEC is increased in type 1 diabetes independent of HDLcholesterol, and that this increase is contributed to particularly by ABCA1-independent CEC.

We demonstrated sex-specific differences in HDL biology in type 1 diabetes with preservation of HDL particle concentration and increased ABCA1-independent efflux evident in men but not in women relative to non-diabetic participants. In contrast, there was a profound reduction in the concentration of small HDL particles in women with type 1 diabetes compared with non-diabetic women, with no effect evident in men. Notably, however, division of type 1 diabetes participants by sex resulted in relatively small cohorts of 40 men and 60 women; these observations will require verification and further analysis in larger type 1 diabetes populations.

The observed increase in CEC in type 1 diabetes occurred in parallel with increased HDL-cholesterol concentration, HDL particle size and large HDL particle concentration. Although not previously demonstrated in populations with type 1 diabetes, the association between larger HDL particles and total CEC has been reported in older adults [23] and perimenopausal women [24]. In the current study, consistent with increased particle size, the increase in total CEC was predominantly attributable to increased $\mathrm{ABCA} 1$-independent CEC [11], with a lesser increase in ABCA1-dependent CEC. Interestingly, despite the reduction in concentration of smalland medium-sized HDL particles in type 1 diabetes, ABCA1dependent $C E C$ was enhanced relative to non-diabetic participants. ABCA1-dependent efflux typically correlates with 
lipid-poor pre- $\beta$-1 HDL and not with large HDL particles [25] and thus these findings were unexpected; they indicate that the larger-sized HDL particles in type 1 diabetes might facilitate greater storage of non-esterified cholesterol, causing this unexpected change in association between ABCA1dependent efflux and particle size. It is also important to note that NMR is limited in its ability to detect lipid-poor pre- $\beta-1$ HDL [26] as it specifically measures cholesterol on lipoprotein particles. These lipid-poor pre- $\beta-1$ HDL particles are the primary acceptor of ABCA1-dependent CEC [27] and thus we potentially did not capture the true association between ABCA1-dependent CEC and small HDL particles in the current study.

We previously demonstrated increased association of the acute-phase inflammatory protein serum amyloid A (SAA) on HDL2 and HDL3 subfractions in type 1 diabetes [28]. Enrichment of HDL with SAA has been reported to reduce CEC [29, 30], particularly in an acute-phase setting [31, 32], and impair the anti-inflammatory properties of the HDL particles [33]. The degree of enrichment of HDL with SAA during the acute-phase response ( 1000 -fold) [34], however, is considerably higher than that seen in type 1 diabetes $(\sim 1.7$ fold) and therefore differences observed in type 1 diabetes might not be clinically relevant.

The observed increase in HDL particle size in type 1 diabetes has possible important implications for RCT through the lymphatic vasculature [35]. The level and particle size of HDL within the interstitial fluid that bathes peripheral cells is arguably more important for RCT than the size of particles within serum. Approximately $50 \%$ of all ApoA-I circulates within the interstitial fluid of peripheral organs [36], where pre- $\beta$ particles predominate and CETP activity is low relative to plasma [36]. Recent findings by Apro et al have demonstrated that the CEC of HDL derived from interstitial fluid was significantly lower than that derived from plasma [37]. Furthermore, HDL CEC was significantly reduced in individuals with type 2 diabetes relative to healthy controls and this effect was more pronounced in HDL from interstitial fluid compared with plasma [37]. A limitation of the current study is that we have specifically measured serum CEC and not interstitial fluid CEC. Thus, while serum CEC is enhanced in type 1 diabetes, the shift towards larger particle size might in fact reduce access to the interstitial fluid and reduce this important route of RCT. In particular, the remarkable reduction in small HDL particle number in women may lead to loss of cholesterol flux through the lymphatic RCT pathway. This requires further investigation as it potentially contributes to residual cardiovascular risk in individuals with type 1 diabetes.

The current study demonstrates both potentially protective (increased HDL particle size and CEC) and harmful (reduced HDL particle number) effects of type 1 diabetes on HDL biology. Similar to CEC, measuring the number of HDL particles by NMR has been shown in numerous trials (including the Veterans Affairs High-Density Lipoprotein Intervention Trial [VA-HIT] and the primary prevention Multiple Risk Factor Intervention Trial [MRFIT]) to be a better predictor of future cardiovascular events than circulating HDL-cholesterol or ApoA-I levels [38, 39] (reviewed in reference [40]). Recent subanalysis of findings from Justification for the Use of Statins in Prevention: an Intervention Trial Evaluating Rosuvastatin (JUPITER) has suggested that NMR-measured HDL particle number is superior to CEC in predicting CVD events [41]. Levels of large HDL particle subpopulations, as determined by NMR, are inversely associated with CVD risk while levels of small HDL particles are often positively correlated with risk [40, 42]. Circulating levels of large $\alpha-1$ HDL, as determined by 2D gel electrophoresis, are also associated with reduced cardiovascular risk while levels of small HDL particles are elevated in individuals with CHD [43-45]. The Epidemiology of Diabetes Complications (EDC) study demonstrated prospectively that HDL particle size could predict the development of cardiovascular events in type 1 diabetes [46]. Similar to our findings, Colhoun et al demonstrated more large HDL particles and fewer small HDL particles in type 1 diabetes, and greater mean HDL particle size [47]; however, they did not find a relationship between particle size and computer tomography-defined coronary artery calcification and in the current study we did not demonstrate associations between any components of HDL structure and function and CIMT.

We observed a positive correlation between both ABCA1dependent and ABCA1-independent CEC and CIMT, although these effects did not persist in multivariable analysis. While this was unexpected it is noteworthy that the study participants were relatively young (mean age 37 years) and predominantly female $(60 \%)$ with low lesion burden. Furthermore, the association between CEC and CVD is controversial. The cornerstone study by Khera et al demonstrated an association between HDL CEC and CVD burden in an older population (mean age $57 \pm 9$ years) with angiographically confirmed CVD [12]. Khera et al have more recently demonstrated superiority of measuring HDL particle number compared with HDL CEC as a predictor of incident CVD, finding no association between baseline CEC and incident CVD within JUPITER [41]. However, apparently contrary to these findings, $\mathrm{Li}$ et al demonstrated a positive association between CEC and cardiovascular events in non-diabetic individuals (1.66-fold increase in MACE), findings that remained similar (1.85-fold increase) after adjustment for traditional cardiovascular risk factors [48]. Furthermore, in the Multiethnic Study of Atherosclerosis [14], CEC was inversely associated with incident coronary artery disease but positively associated with carotid plaque progression. Taken together, these findings suggest significant complexity in the 
association between CEC and progression of atherosclerosis at different sites. Larger studies including type 1 diabetes participants only are needed to clarify whether and to what extent changes in both particle size and CEC influence atherosclerosis risk in this condition.

An important consideration in future studies will be investigation of the effects of statin treatment and alcohol intake on HDL structure and function in diabetes. Although statin treatment was independently and positively associated with CEC in multivariable analysis, it is difficult to separate this apparent effect from the fact that statins were predominantly used in older male participants, and only in the type 1 diabetes group. Alcohol intake was greater in non-diabetic participants and statistically significant correlations were identified between alcohol intake and certain components of CEC. Limiting analysis, however, to participants from both groups with weekly alcohol intake not greater than ten units did not change our findings. Future studies in larger cohorts will help explore potential contributions of statins and alcohol to HDL structure and function in diabetes.

In conclusion, we have demonstrated potentially important differences in CEC, particularly its ABCA1independent component, in type 1 diabetes. These differences remain statistically significant following adjustment for differences in HDL-cholesterol concentration and HDL particle size and concentration. Future studies are needed to determine the mechanism underlying these differences and their role in the atherosclerotic complications of type 1 diabetes.

Acknowledgements Some of the data reported in this manuscript were presented at the Irish Endocrine Society AGM in 2018.

Data availability Data are available on request from the authors.

Funding sources This work was funded by a grant from The Meath Foundation and by a legacy from the estate of the late Robert George Davies.

Authors' relationships and activities The authors declare that there are no relevant relationships or activities that might bias, or be perceived to bias, their work.

Contribution statement MOA co-designed the study, recruited the participants, measured CIMT, analysed clinical data and prepared the first draft of paper. FCM and JG co-designed the study: JG managed the clinical recruitment and CIMT analysis; and FCM led the laboratorybased analysis of HDL. REB performed the HDL function assays, analysed data, prepared figures and co-wrote the first draft of the paper. WG, AP, AG, IF, MS, KSA, AM and KM were involved in study design, recruitment of participants into the study, clinical measurements of CIMT and reviewed and edited the final manuscript. GB collaborated on study design, sample analysis, data acquisition and reviewed the manuscript. RS planned the re-analysis of data for the revised manuscript, oversaw data analysis and presentation, and description of the new statistical methods and results throughout the text, and helped draft the final manuscript. FCM and JG co-edited the final paper and all authors approved the final draft. JG is responsible for the integrity of the work as a whole.

\section{References}

1. Lind M, Svensson AM, Rosengren A (2015) Glycemic control and excess mortality in type 1 diabetes. N Engl J Med 372(9):880-881. https://doi.org/10.1056/NEJMc1415677

2. Livingstone SJ, Levin D, Looker HC et al (2015) Estimated life expectancy in a Scottish cohort with type 1 diabetes, 2008-2010. JAMA 313(1):37-44. https://doi.org/10.1001/jama.2014.16425

3. de Ferranti SD, de Boer IH, Fonseca V et al (2014) Type 1 diabetes mellitus and cardiovascular disease: a scientific statement from the American Heart Association and American Diabetes Association. Circulation 130(13):1110-1130. https://doi.org/10.1161/CIR. 0000000000000034

4. Miller GJ, Miller NE (1975) Plasma-high-density-lipoprotein concentration and development of ischaemic heart-disease. Lancet 1(7897):16-19. https://doi.org/10.1016/s0140-6736(75)92376-4

5. Barter PJ, Caulfield M, Eriksson M et al (2007) Effects of torcetrapib in patients at high risk for coronary events. N Engl J Med 357(21):2109-2122. https://doi.org/10.1056/ NEJMoa0706628

6. Schwartz GG, Olsson AG, Abt M et al (2012) Effects of dalcetrapib in patients with a recent acute coronary syndrome. N Engl J Med 367(22):2089-2099. https://doi.org/10.1056/NEJMoa1206797

7. Navab M, Reddy ST, Van Lenten BJ, Fogelman AM (2011) HDL and cardiovascular disease: atherogenic and atheroprotective mechanisms. Nat Rev Cardiol 8(4):222-232. https://doi.org/10.1038/ nrcardio.2010.222

8. Rader DJ (2003) Regulation of reverse cholesterol transport and clinical implications. Am J Cardiol 92(4A):42J-49J. https://doi. org/10.1016/s0002-9149(03)00615-5

9. Back SS, Kim J, Choi D, Lee ES, Choi SY, Han K (2013) Cooperative transcriptional activation of ATP-binding cassette sterol transporters $\mathrm{ABCG} 5$ and $\mathrm{ABCG} 8$ genes by nuclear receptors including Liver-X-Receptor. BMB Rep 46(6):322-327. https://doi. org/10.5483/BMBRep.2013.46.6.246

10. Noe J, Kullak-Ublick GA, Jochum W et al (2005) Impaired expression and function of the bile salt export pump due to three novel ABCB11 mutations in intrahepatic cholestasis. J Hepatol 43(3): 536-543. https://doi.org/10.1016/j.jhep.2005.05.020

11. Yancey PG, Bortnick AE, Kellner-Weibel G, de la Llera-Moya M, Phillips MC, Rothblat GH (2003) Importance of different pathways of cellular cholesterol efflux. Arterioscler Thromb Vasc Biol 23(5):712-719. https://doi.org/10.1161/01.ATV. 0000057572.97137.DD

12. Khera AV, Cuchel M, de la Llera-Moya M et al (2011) Cholesterol efflux capacity, high-density lipoprotein function, and atherosclerosis. N Engl J Med 364(2):127-135. https://doi.org/10.1056/ NEJMoa1001689

13. Rohatgi A, Khera A, Berry JD et al (2014) HDL cholesterol efflux capacity and incident cardiovascular events. N Engl J Med 371(25): 2383-2393. https://doi.org/10.1056/NEJMoa1409065

14. Shea S, Stein JH, Jorgensen NW et al (2019) Cholesterol mass efflux capacity, incident cardiovascular disease, and progression of carotid plaque. Arterioscler Thromb Vasc Biol 39(1):89-96. https://doi.org/10.1161/ATVBAHA.118.311366

15. Ganjali S, Dallinga-Thie GM, Simental-Mendía LE, Banach M, Pirro M, Sahebkar A (2017) HDL functionality in type 1 diabetes. Atherosclerosis 267:99-109. https://doi.org/10.1016/j. atherosclerosis.2017.10.018

16. Costacou T, Evans RW, Orchard TJ (2011) High-density lipoprotein cholesterol in diabetes: is higher always better? J Clin Lipidol 5(5):387-394. https://doi.org/10.1016/j.jacl.2011.06.011

17. Jeyarajah EJ, Cromwell WC, Otvos JD (2006) Lipoprotein particle analysis by nuclear magnetic resonance spectroscopy. Clin Lab Med 26(4):847-870. https://doi.org/10.1016/j.cl1.2006.07.006 
18. Adrees M, Gibney J, El-Saeity N, Boran G (2009) Effects of 18 months of L-T4 replacement in women with subclinical hypothyroidism. Clin Endocrinol 71(2):298-303. https://doi.org/10.1111/j. 1365-2265.2008.03509.x

19. Daffu G, Shen X, Senatus L et al (2015) RAGE suppresses ABCG1-mediated macrophage cholesterol efflux in diabetes. Diabetes 64(12):4046-4060. https://doi.org/10.2337/db15-0575

20. Mauldin JP, Nagelin MH, Wojcik AJ et al (2008) Reduced expression of ATP-binding cassette transporter G1 increases cholesterol accumulation in macrophages of patients with type 2 diabetes mellitus. Circulation 117(21):2785-2792. https://doi.org/10.1161/ CIRCULATIONAHA.107.741314

21. de Vries R, Kerstens MN, Sluiter WJ, Groen AK, van Tol A, Dullaart RP (2005) Cellular cholesterol efflux to plasma from moderately hypercholesterolaemic type 1 diabetic patients is enhanced, and is unaffected by simvastatin treatment. Diabetologia 48(6):1105-1113. https://doi.org/10.1007/s00125005-1760-0

22. Manjunatha S, Distelmaier K, Dasari S, Carter RE, Kudva YC, Nair KS (2016) Functional and proteomic alterations of plasma high density lipoproteins in type 1 diabetes mellitus. Metabolism 65(9):1421-1431. https://doi.org/10.1016/j.metabol.2016.06.008

23. Mutharasan RK, Thaxton CS, Berry J et al (2017) HDL efflux capacity, HDL particle size, and high-risk carotid atherosclerosis in a cohort of asymptomatic older adults: the Chicago Healthy Aging Study. J Lipid Res 58(3):600-606. https://doi.org/10.1194/ jlr.P069039

24. El Khoudary SR, Hutchins PM, Matthews KA et al (2016) Cholesterol efflux capacity and subclasses of HDL particles in healthy women transitioning through menopause. J Clin Endocrinol Metab 101(9):3419-3428. https://doi.org/10.1210/jc. 2016-2144

25. de la Llera-Moya M, Drazul-Schrader D, Asztalos BF, Cuchel M, Rader DJ, Rothblat GH (2010) The ability to promote efflux via ABCA1 determines the capacity of serum specimens with similar high-density lipoprotein cholesterol to remove cholesterol from macrophages. Arterioscler Thromb Vasc Biol 30(4):796-801. https://doi.org/10.1161/ATVBAHA.109.199158

26. Rosenson RS, Brewer HB Jr, Chapman MJ et al (2011) HDL measures, particle heterogeneity, proposed nomenclature, and relation to atherosclerotic cardiovascular events. Clin Chem 57(3):392410. https://doi.org/10.1373/clinchem.2010.155333

27. Favari E, Lee M, Calabresi L et al (2004) Depletion of pre- $\beta$-high density lipoprotein by human chymase impairs ATP-binding cassette transporter A1- but not scavenger receptor class B type Imediated lipid efflux to high density lipoprotein. J Biol Chem 279(11):9930-9936. https://doi.org/10.1074/jbc.M312476200

28. McEneny J, Daniels JA, McGowan A et al (2015) A cross-sectional study demonstrating increased serum amyloid a related inflammation in high-density lipoproteins from subjects with type 1 diabetes mellitus and how this association was augmented by poor glycaemic control. J Diabetes Res 2015:351601. https://doi.org/ 10.1155/2015/351601

29. Vaisar T, Tang C, Babenko I et al (2015) Inflammatory remodeling of the HDL proteome impairs cholesterol efflux capacity. J Lipid Res 56(8):1519-1530. https://doi.org/10.1194/jlr.M059089

30. Banka CL, Yuan T, de Beer MC, Kindy M, Curtiss LK, de Beer FC (1995) Serum amyloid A (SAA): influence on HDL-mediated cellular cholesterol efflux. J Lipid Res 36(5):1058-1065

31. de la Llera Moya M, McGillicuddy FC, Hinkle CC et al (2012) Inflammation modulates human HDL composition and function in vivo. Atherosclerosis 222(2):390-394. https://doi.org/10.1016/j. atherosclerosis.2012.02.032

32. McGillicuddy FC, de la Llera Moya M, Hinkle CC et al (2009) Inflammation impairs reverse cholesterol transport in vivo.
Circulation 119(8):1135-1145. https://doi.org/10.1161/ CIRCULATIONAHA.108.810721

33. Han CY, Tang C, Guevara ME et al (2016) Serum amyloid A impairs the antiinflammatory properties of HDL. J Clin Invest 126(2):796. https://doi.org/10.1172/JCI86401

34. Coetzee GA, Strachan AF, van der Westhuyzen DR, Hoppe HC, Jeenah MS, de Beer FC (1986) Serum amyloid A-containing human high density lipoprotein 3. Density, size, and apolipoprotein composition. J Biol Chem 261(21):9644-9651

35. Martel C, Li W, Fulp B et al (2013) Lymphatic vasculature mediates macrophage reverse cholesterol transport in mice. J Clin Invest 123(4):1571-1579. https://doi.org/10.1172/JCI63685

36. Miller NE, Olszewski WL, Hattori H et al (2013) Lipoprotein remodeling generates lipid-poor apolipoprotein A-I particles in human interstitial fluid. Am J Physiol Endocrinol Metab 304(3): E321-E328. https://doi.org/10.1152/ajpendo.00324.2012

37. Apro J, Tietge UJ, Dikkers A, Parini P, Angelin B, Rudling M (2016) Impaired cholesterol efflux capacity of high-density lipoprotein isolated from interstitial fluid in type 2 diabetes mellitus-brief report. Arterioscler Thromb Vasc Biol 36(5):787-791. https://doi. org/10.1161/ATVBAHA.116.307385

38. Otvos JD, Collins D, Freedman DS et al (2006) Low-density lipoprotein and high-density lipoprotein particle subclasses predict coronary events and are favorably changed by gemfibrozil therapy in the Veterans Affairs High-Density Lipoprotein Intervention Trial. Circulation 113(12):1556-1563. https://doi.org/10.1161/ CIRCULATIONAHA.105.565135

39. Kuller LH, Grandits G, Cohen JD, Neaton JD, Prineas R, Multiple Risk Factor Intervention Trial Research Group (2007) Lipoprotein particles, insulin, adiponectin, C-reactive protein and risk of coronary heart disease among men with metabolic syndrome. Atherosclerosis 195(1):122-128. https://doi.org/10.1016/j. atherosclerosis.2006.09.001

40. Kontush A (2015) HDL particle number and size as predictors of cardiovascular disease. Front Pharmacol 6:218. https://doi.org/10. 3389/fphar.2015.00218

41. Khera AV, Demler OV, Adelman SJ et al (2017) Cholesterol efflux capacity, high-density lipoprotein particle number, and incident cardiovascular events: an analysis from the JUPITER trial (Justification for the Use of Statins in Prevention: An Intervention Trial Evaluating Rosuvastatin). Circulation 135(25):2494-2504. https://doi.org/10.1161/CIRCULATIONAHA.116.025678

42. Kuller L, Arnold A, Tracy R et al (2002) Nuclear magnetic resonance spectroscopy of lipoproteins and risk of coronary heart disease in the cardiovascular health study. Arterioscler Thromb Vasc Biol 22(7):1175-1180. https://doi.org/10.1161/01.atv. 0000022015.97341.3a

43. Asztalos BF, Schaefer EJ (2003) HDL in atherosclerosis: actor or bystander? Atheroscler Suppl 4(1):21-29. https://doi.org/10.1016/ s1567-5688(03)00006-0

44. Asztalos BF, Schaefer EJ (2003) High-density lipoprotein subpopulations in pathologic conditions. Am J Cardiol 91(7A):12E-17E. https://doi.org/10.1016/s0002-9149(02)03383-0

45. Lamon-Fava S, Herrington DM, Reboussin DM et al (2008) Plasma levels of HDL subpopulations and remnant lipoproteins predict the extent of angiographically-defined coronary artery disease in postmenopausal women. Arterioscler Thromb Vasc Biol 28(3):575579. https://doi.org/10.1161/ATVBAHA.107.157123

46. Soedamah-Muthu SS, Chang YF, Otvos J, Evans RW, Orchard TJ, Pittsburgh Epidemiology of Diabetes Complications Study (2003) Lipoprotein subclass measurements by nuclear magnetic resonance spectroscopy improve the prediction of coronary artery disease in Type 1 diabetes. A prospective report from the Pittsburgh Epidemiology of Diabetes Complications Study. Diabetologia 46(5):674-682. https://doi.org/10.1007/s00125-003-1094-8 
47. Colhoun HM, Otvos JD, Rubens MB, Taskinen MR, Underwood SR, Fuller JH (2002) Lipoprotein subclasses and particle sizes and their relationship with coronary artery calcification in men and women with and without type 1 diabetes. Diabetes 51(6):19491956. https://doi.org/10.2337/diabetes.51.6.1949

48. Li X-M, Tang WHW, Mosior MK et al (2013) Paradoxical association of enhanced cholesterol efflux with increased incident cardiovascular risks. Arterioscler Thromb Vasc Biol 33(7):16961705. https://doi.org/10.1161/ATVBAHA.113.301373

Publisher's note Springer Nature remains neutral with regard to jurisdictional claims in published maps and institutional affiliations. 